\title{
O Uso do Brinquedo Terapêutico na Vacinação
}

\author{
The use of therapeutic toys in vaccination
}

\section{El uso de juguetes terapéuticos en vacunación}

\section{Luany Mazoni dos Santos ${ }^{1 *}$, Janaínna Sther Leite Godinho silva ${ }^{2}$, Alessandra da Silva Souza ${ }^{3}$, Eliara Adelino da Silva ${ }^{4}$, Carolina de Lourdes Julião Vieira ${ }^{5}$, Lília Marques Simões Rodrigues Rodrigues ${ }^{6}$ \\ Como citar esse artigo. dos Santos, LM; Silva, JSLG; Souza, AS; da Silva, EA; \\ Resumo} Vieira, CLJ; Rodrigues, LMSR. O Uso do Brinquedo Terapêutico na Vacinação Revista Pró-UniverSUS. 2021 Jul./ Dez.; 12 (2)SUPLEMENTO: 85 - 89

Este estudo tem como objetivo descrever a utilização os brinquedos terapêuticos durante o processo de vacinação. Trata-se de um estudo qualitativo exploratório descritivo que foi realizado através de uma revisão integrativa de literatura. $\mathrm{O}$ estudo foi realizado mediante a buscas nas bases de dados BIREME, LILACS, MEDLINE e SCIELO, usando os descritores Enfermagem, Brinquedo Terapêutico, Saúde Pública e Vacinação com estudos publicados entre 2011 a 2019. Foi evidenciado durante o estudo que apesar das dificuldades na implantação da prática na vacinação, o enfermeiro pediatra deve utilizar o brinquedo terapêutico no cotidiano de trabalho e orientar a equipe de enfermagem que cuida diretamente da criança a técnica correta para que tenhamos bons resultados, onde a criança entenda o procedimento que está sendo realizado e possa perder o pavor que os mesmos tem na hora da vacinação.

Palavras-chave: Brinquedo Terapêutico; Enfermagem; Saúde Pública; Vacina

\begin{abstract}
This study aims to describe the use of therapeutic toys during the vaccination process. This is a descriptive exploratory qualitative study that was carried out through an integrative literature review. The study was carried out by searching the BIREME, LILACS, MEDLINE and SCIELO databases, using the descriptors Nursing, Therapeutic Toy, Public Health and Vaccination with studies published between 2011 and 2019. It was evidenced during the study that despite the difficulties in implantation of the practice in vaccination, the pediatric nurse must use the therapeutic toy in the daily work and guide the nursing team that directly takes care of the child the correct technique so that we have good results, where the child understands the procedure being performed and may lose the dread they have at the time of vaccination.

Keywords: Therapeutic Toy; Nursing; Public Health; Vaccine.
\end{abstract}

estudio tiene como objetivo describir el uso de juguetes terapéuticos durante el proceso de vacunación. Se trata de estudio cualitativo exploratorio descriptivo que se realizó mediante una revisión integradora de la literatura. El estudio se realizó mediante la búsqueda en las bases de datos BIREME, LILACS, MEDLINE y SCIELO, utilizando los descriptores Enfermería, Juguete Terapéutico, Salud Pública y Vacunación con estudios publicados entre 2011 y 2019. Se evidenció durante el estudio que, a pesar de las dificultades de implantación de la práctica en vacunación, la enfermera pediátrica debe utilizar el juguete terapéutico en el trabajo diario y orientar al equipo de enfermería que atiende directamente al niño la técnica correcta para que tengamos buenos resultados, donde el niño comprenda el procedimiento que se está realizando y pueda perder el pavor que tienen al momento de la vacunación.

Palabras clave: Juguete Terapéutico; Enfermería; Salud Pública; Vacuna.

Afiliação dos autores:

${ }^{1}$ Acadêmica do Curso de Enfermagem, Universidade de Vassouras. RJ, Brasil. E-mail: luannymazoni.luuh@gmail.com ORCID: https://orcid.org/0000-0003-2479-0847

${ }^{2}$ Mestre. Docente do Curso de Enfermagem, Universidade de Vassouras. RJ, Brasil. E-mail: jjasther@gmail.com ORCID: https://orcid.org/0000-0002-8308-2093

${ }^{3}$ Mestre. Docente do Curso de Enfermagem, Universidade de Vassouras. RJ, Brasil. E-mail: alesouza22@yahoo.com.br ORCID: https://orcid.org/0000-0002-9009-9774

${ }^{4}$ Mestre. Terapeuta Holística. Docente do Curso de Enfermagem, Universidade de Vassouras. RJ, Brasil. E-mail: eliaraadelinos@uol.com.br ORCID: https://orcid.org/0000-0003-

4387-6662

${ }^{5}$ Doutora. Docente do Curso de Enfermagem, Universidade de Vassouras. RJ, Brasil. E-mail: caroljuliaovieira@gmail.com ORCID: http://orcid.org/0000-0002-5785-5988

${ }^{6}$ Mestre. Coordenadora e Docente do Curso de Enfermagem, Universidade de Vassouras. RJ, Brasil. E-mail: liliasrodrigues21@gmail.com ORCID: https://orcid.org/0000-0003-

$2979-6316$

* Email de correspondencia: luannymazoni.luuh@gmail.com

Recebido em: 21/01/21. Aceito em: 18/05/21 


\section{Introdução}

A brincadeira é muito importante na infância, pois por meio dela as crianças podem entender, aprender e se expressar, o que ajuda no seu desenvolvimento físico, motor e emocional, pois ao contrário dos adultos, as crianças ainda não sabem dizer o que sentem. ${ }^{1}$

É estabelecido pelo artigo 16, do Estatuto da Criança e do Adolescente que toda criança tem o direito à liberdade e ao respeito. Compreendendo os aspectos de brincar e se divertir, sendo o direito de ser criança, ajudando no desenvolvimento infantil. ${ }^{2}$

A brincadeira pode ser dividida em dois tipos: Entretenimento: definido como uma atividade não estruturalnaqual as crianças participam espontaneamente para se divertir e promover a interação entre as crianças. Terapêutica: definida como atividades organizadas, realizadas por profissionais bem treinados que aplicam brinquedos terapêticos, com o objetivo de promover a saúde física e mental de crianças em idade anormal. ${ }^{1}$

Alguns procedimentos invasivos (como a vacinação) acabam angustiando as crianças, tornandoas eventualmente irritáveis, agressivas e traumatizadas. Em suma, devido aos traumas causados pelas crianças ao longo dos anos, os profissionais de saúde devem tratar as crianças com maior segurança. Os brinquedos terapêuticos auxiliam a criança a entendero procedimento a ser realizado, auxilia a diminuir o nervosismo durante a vacinação, o medo e a ansiedade. ${ }^{4}$

Atuar como enfermeiro em todas as fases do desenvolvimento humano é extremamente desafiador, pois énecessário aprofundaros sentidos na fase específica da vida em que o paciente se descobre para compreender as necessidades humanas do paciente. Quando cuidamos de crianças pré-escolares (3-6 anos), esse desafio será ainda mais severo, pois elas tendem a mostrar fantasia e egocentrismo, além de sua compreensão pouco clara dos limites físicos e da anatomia pensei em. ${ }^{1}$

O brinquedo terapêutico é o brinquedo básico em pediatria, eventualmente, o profissional de saúde poderá utilizá-lo em procedimentos invasivos, para melhor aceitação da criança, pois se não houver brinquedo terapêutico a criança formará obstáculos, o que eventualmente dificultará a realização de determinados procedimentos. Esta ferramenta deve ser usada quando as crianças têm dificuldade em compreender ou enfrentam certas ameaças (como vacinas e outros procedimentos invasivos). ${ }^{3}$

Outro benefício do cuidado com o brinquedo é que ele fortalece o relacionamento com a criança e a família. A relação interativa baseada na confiança e segurança no jogo faz com que as crianças/família se sintam mais à vontade e expressem suas dúvidas durante a internação. Isso permitirá que os enfermeiros entendam melhor a criança e as razões de determinados comportamentos. ${ }^{4}$

A Resolução no 546/2017 estipula a utilização de brinquedos terapêuticos nos quais o enfermeiro pode utilizar os brinquedos para puericultura. ${ }^{5}$

Este estudo tem como objetivo descrever o uso de brinquedos terapêuticos durante a vacinação, com intuito de mostrar uma forma mais extrovertida para a criança no seu momento de pânico quando se trata de vacina. Através dos brinquedos terapêuticos a criança poderá aprender o procedimento com mais clareza e sua importância, com isso desde pequeno vai aprender como é importante tomar todas as vacinas e não se preocupar quando for a hora de realizar o procedimento.

O brinquedo terapêutico deve ser usado assim que a criança chega para poder se vacinar, o profissional devera pegar o brinquedo e demostrar para a criança como vai ser realizado o procedimento e explicando cada passinho e como é importante o que está sendo realizado, poderá também pedir a ajuda da criança para manusear o brinquedo, deixando-a menos tensa e ajudando a descontrair um pouco.

Desta forma o brinquedo vai ajudar a criança a entender o procedimento que é a vacinação e vai amenizar o sofrimento que os pequeninos têm quando se fala em vacina.

Com base no que foi apresentado a questão que norteou o estudo foi: Como o brinquedo terapêutico é utilizado na prática da vacinação?

Diante do exposto o objetivo do estudo foi verificar o Uso do Brinquedo Terapêutico na Vacinação a partir da literatura científica.

\section{Metodologia}

Tratou-se de um estudo qualitativo, exploratório descritivo que foi realizado através de uma revisão integrativa de literatura.

A revisão integrativa da literatura é um método que visa estudar de forma abrangente os resultados da pesquisa de um determinado tópico ou questão de forma sistemática, ordenada e abrangente. Dessa forma, os revisores/pesquisadores podem projetar cuidadosamente revisões abrangentes com diferentes propósitos e podem conduzir análises metodológicas sobre definições conceituais, revisões teóricas ou pesquisas sobre tópicos específicos. ${ }^{6}$

Além de fundir uma ampla gama de finalidades, também incorpora dados da literatura teórica e empírica: definições de conceitos, revisões de teorias e evidências e análise de questões metodológicas em tópicos específicos. Um grande número de amostras $\mathrm{e}$ um grande número de sugestões devem produzir um panorama consistente e compreensível de conceitos, teorias ou questões de saúde complexas relacionadas à enfermagem. ${ }^{7}$ 
A pesquisa qualitativa desenvolveu reflexão crítica e acumulou conhecimento, é necessário que o pesquisador deixe de usar os padrões implícitos para avaliar e orientar a pesquisa, ou seja, eles não são mais implícitos ou de difícil compreensão ou compreensão dos padrões para adotá-los mais. Padrões claros e processos de investigação permitem que a pesquisa seja compreendida e repetida. ${ }^{8}$

O estudo foi iniciado em setembro de 2020 à dezembro de 2020 através de buscas nas bases de dados Bireme, MEDLine, Sciello e LILACS usando os descritores Enfermagem, Brinquedo Terapêutico, Saúde Pública e Vacinação. O tipo de pesquisa foi uma análise documental integrativa, e a pesquisa foi lançada entre 2011 e2019, os artigos foram relacionados a tema, artigos publicados no idioma português, inglês e espanhol. As buscas ocorreram por meio das junções "Enfermagem and Brinquedo Terapêutico and Vacinação" "Nursing and Therapeutic Toy and Vaccination", obteve-se um resultado de 3 artigos, dentre os quais foram selecionados apenas 1 artigo que estava relacionado com o tema após a leitura do título e resumo. Os outros 2 (dois) foram descartados pelos motivos: por não atenderem o objetivo da pesquisa. Devido ao número insuficiente de artigos, se fez necessário uma nova pesquisa. Para um novo levantamento de dados foram relacionados os seguintes descritores: "Educação em enfermagem and Jogos e brinquedos and Enfermagem pediátrica" "Nursing Education and Games and Toys and Pediatric Nursing no banco de dados citado anteriormente no qual foram encontrados 111 artigos, dentre esses artigos foi aplicado filtro "texto completo", base de dados "LILACS, BIREME, MEDLINE, SCIELO", idioma "Português, Inglês e Espanhol", e assim reduziu para 91 artigos, após a redução foram selecionados 5 artigos e os demais foram descartados pelos motivos: 2 (dois) por estar repetido e 84 (oitenta e quatro) por não atenderem o objetivo da pesquisa. Devido ao número insuficiente de artigos, se fez necessário uma nova pesquisa. Para um novo levantamento de dados foram relacionados os seguintes descritores: "Enfermagem and Jogos e Brinquedos and Pediatria" "Nursing and Games and Toys and Pediatric", obteve-se um resultado de 16 artigos, dentre os quais foram selecionados apenas 4 artigos. Os demais foram descartados pelos motivos: por não atenderem o objetivo da pesquisa.

Foram selecionados para esta pesquisa 10 artigos compondo o conteúdo do presente estudo.

\section{Resultados e Discussão}

Observando a área de conhecimento do periódico, constatou-se que as publicações analisadas na maioria dos estudos envolviam o uso de brinquedos terapêuticos na atenção básica a saúde foi publicada na Revista de
Enfermagem UFPE totalizando em 04 artigos conforme a tabela abaixo.

Tabela 1. Números de artigos.

\begin{tabular}{|l|c|}
\hline \multicolumn{1}{|c|}{ PERIÓDICOS } & $\begin{array}{c}\text { NÚMERO DE } \\
\text { ARTIGOS }\end{array}$ \\
\hline Revista Brasileira de Enfermagem & 03 \\
\hline Revista Cuidarte & 02 \\
\hline Revista de Enfermagem UFPE - Online & 04 \\
\hline Revista Baiana & 01 \\
\hline Total & 10 \\
\hline
\end{tabular}

Fonte: Dados da própria pesquisa.

A partir dos dados coletados percebi que as crianças que têm abordagem com o brinquedo terapêutico ficam mais tranquilas e relaxadas na hora do procedimento, aceitando melhor a conduta. E as crianças que não têm acesso ao brinquedo terapêutico acabam dificultando o procedimento, pois as mesmas apresentam comportamentos de resistência. ${ }^{9}$

O foco do uso do brinquedo terapêutico é ajudar a criança a aliviar a ansiedade vivida durante a vacinação, para que ela exponha seus sentimentos (medo, ansiedade, tensão e necessidades), de modo a absorver o método de tratamento feito pela equipe de enfermagem sobre o procedimento, visto que a categoria profissional está mais próxima da criança durante o momento da aplicação. ${ }^{10}$

Quanto mais a criança entender o que vai acontecer com ela, menos ela terá medo. Os brinquedos podem ajudá-la a lidar com a realidade e permitir que ela experimente o processo doloroso de uma maneira extrovertida. ${ }^{9}$

Quando o brinquedo terapêutico é utilizado entre a criança e o profissional de saúde a comunicação entre ambos acaba se tornando muito mais lucrativa e extrovertida, com isso, acaba criando um vínculo com o pequenino facilitando na hora do procedimento. ${ }^{11}$

Os pais ao participarem junto com o(s) filho(s) no momento do brinquedo terapêutico relata que o brincar antes do procedimento invasivo alivia a tensão e a ansiedade que é gerada na criança quando os mesmos sabem que vão precisar se vacinar, com isso, o(s) filho(s) ficam mais relaxados e muito mais compreensivos. ${ }^{12}$

Osacompanhantesaovivenciaremessaexperiência percebem os pontos positivos que o brinquedo transfere para a criança, ajudando o emocional que se encontra um pouco abalado, ajudando criar uma relação melhor 
entre profissional e a criança. ${ }^{13}$

Ao notar que seu(s) filho(s) ficam mais calmos com a utilização do brinquedo na hora do preparo do procedimento, os pais acabam ficando mais tranquilos e mais seguros, fazendo com que o ambiente fique com um aspecto menos tenso e que a(s) criança(s) se sintam mais seguras. ${ }^{14}$

Ao usar o brinquedo é notório uma melhora no quadro emocional da criança, com isso, os profissionais acabam promovendo uma relação de confiança entre os familiares e também entre os pequeninos. ${ }^{15}$

Aplicando essa técnica a(s) criança(s) perdem o medo que tem do profissional de enfermagem, pois ela(s) sempre associa os profissionais aos procedimentos que lhe causaram dor. ${ }^{16}$

É importante ressaltar o uso do brinquedo nos cursos de Enfermagem, facilitando o contato aos acadêmicos para praticarem essa técnica e assim tornar uma assistência mais humanizada. ${ }^{17}$

Para a enfermagem o brinquedo terapêutico é interessante pois proporciona uma vida muito mais próxima do normal da criança, trazendo um bem-estar e promovendo uma relação saudável entre profissional e o paciente. ${ }^{18}$

O fator e facilitador da utilização do brinquedo terapêutico é que os profissionais compreendem a importância do brincar com recurso positivo na melhora clínica da criança. ${ }^{16}$

Integrandoasações paraqualificação daassistência de enfermagem ao atender ao paciente pediátrico no procedimento de vacinação o brinquedo terapêutico é usado como uma estratégia muito eficaz para alcançar uma aproximação saudável com a criança. ${ }^{15}$

Mesmo podendo compreender todos os benefícios que essa técnica traz para o quadro clínico e emocional da criança, o manuseio desta técnica ainda não é muito utilizado pelas instituições de saúde. ${ }^{10}$

Foi percebido durante a análise dos artigos desse estudo dificuldades encontradas pela equipe de enfermagem ao utilizar o brinquedo terapêutico pois muitos profissionais relatam também que por conta de ter muito trabalho não conseguem manusear este tipo de técnica por falta de tempo, pois sua vida profissional acaba sendo um pouco corrida. ${ }^{1}$.

\section{Conclusão}

O período da vacinação é extremamente difícil para a criança. No primeiro momento ele se depara com o medo e a ansiedade e em seguida ele se depara com a tensão e a dor no momento da aplicação. Segundo as pesquisas feitas, a utilização de brinquedos terapêuticos durante o processo de tratamento é muito importante, pois está estratégia eficaz pode reduzir a tensão e a dor das crianças durante o processo de vacinação. A eficácia do brinquedo terapêutico é bem alta pois com o brinquedo a criança compreende e perde esse pavor que ao longo do tempo a criança vem adquirindo. $\mathrm{E}$ com a(s) criança(s) perdendo esse medo, logo os pais e acompanhantes acabam criando uma confiança entre os profissionais de saúde, fazendo com que criem um vínculo tanto entre familiares, e entre as crianças, facilitando o atendimento para ambos e tornando a vacinação muito mais extrovertida, sempre visando o bem estar da criança.

Com base no que foi debatido neste estudo considero que os objetivos da pesquisa foram contemplados, descrevendo a utilização do brinquedo terapêutico durante o processo de vacinação, a partir da literatura científica, retratando os fatores facilitadores e os fatores que dificultam a sua inserção e com isso contribuindo positivamente na qualidade da prática profissional do enfermeiro durante o procedimento de vacinação da criança.

\section{Referências}

1. Juliani, RML, \& da Silva SA. O uso do brinquedo terapêutico no Processo de Vacinação. Revista Pró-UniverSUS 2019, 10(1), 47-50.

2. Lei n ${ }^{\circ} 8.069$, de 13 de julho de 1990 (BR). Dispõe sobre o Estatuto da Criança e do Adolescente e dá outras providências. Diário Oficial da União [Internet]. 1990 July 13 [cited 208 abr 05]. [acesso em 20 janeiro 2020]. Disponível em: http://www.planalto.gov.br/ccivil_03/leis/18069.htm

3. Cintra, SMP, Silva, CVD, \& Ribeiro, CA. O ensino do brinquedo/ brinquedo terapêutico nos cursos de graduação em enfermagem no Estado de São Paulo. Revista Brasileira de Enfermagem 2006, 59(4), 497-501.

4. Veiga, MA, Sousa, MC, \& Pereira, RS. Enfermagem e o brinquedo terapêutico: vantagens do uso e dificuldades. Rev Eletrôn Atual Saúde 2016, $3(3), 60-6$.

5. COFEN. Resolução no 546, de 09 de maio de 2017. Dispõe sobre a utilização de técnica de brinquedo terapêutico pela Enfermagem. 2017. [acesso em 26 janeiro 2020]. Disponível em: http://www.cofen.gov.br/ resolucao-cofen-no-05462017_52036.html

6. Ercole, FF, Melo, LSD, \& Alcoforado, CLGC. Revisão integrativa versus revisão sistemática. Revista Mineira de Enfermagem 2014, 18(1), $9-12$.

7. Souza, MTD, Silva, MDD, \& Carvalho, RD. Revisão integrativa: o que é e como fazer. Einstein (São Paulo) 2010, 8(1), 102-106.

8. de Paiva, JFG, de Souza, LALM, \& de Mello, SCB. Validade e confiabilidade na pesquisa qualitativa em administração. Revista de Ciências da Administração 2011, 13(31), 190-209.

9. Pontes, JED, Tabet, E, Folkmann, MÁDS, Cunha, MLDR, \& Almeida, FDA. Therapeutic play: preparing the child for the vaccine. Einstein (São Paulo) 2015, 13(2), 238-242.

10. Dantas, FA, da Nóbrega, VM, Pimenta, EAG, \& Collet, N. Brinquedo terapêutico na administração de medicação endovenosa em crianças: estudo exploratório. Online braz. j. nurs.(Online) 2016, 454-465.

11. Souza, LPS, Silva, CCD, Brito, JCAD, Santos, APDO, Fonseca, ADG, Lopes, JR, \& Souza, AAMD. O brinquedo terapêutico e o lúdico na visão da equipe de enfermagem. J Health Sci Inst 2012, 30(4), 354-8.

12. Silva, SRDM, Santos, MCSD, Silva, AMD, Ferreira, FÂ, Freitas, RDSC, Gouveia, MT, \& Santos, READ. Percepção dos acompanhantes das crianças hospitalizadas acerca do brinquedo terapêutico. Rev. enferm. UFPE on line 2018, 2703-2709. 
13. Oliveira, JD, Miranda, MLF, Monteiro, MDFV, \& de Almeida, VDCF.

O brincar e a criança hospitalizada: visão de enfermeiras. Revista Baiana de Enfermagem 2016, 30.

14. Cunha MLR, Brandi, SBGFT, Severino, KG, Almeida, GCF, Campos, PC. Aplicativo para preparo da criança/família na punção venosa: relato de experiência. Rev. Bras. Enferm.

15. Melo, CDF, Almeida, ACACD, \& Araújo Neto, JLD. Brinquedo terapêutico: estratégia de alívio da dor e tensão em crianças com doenças crônicas. Rev. enferm. UFPE on line 2011, 1626-1632.

16. Santos, RDSFV. Brinquedos terapêuticos ajudam crianças a enfrentar o medo e a dor da punção venosa.

17. Lemos, ICS, de Oliveira, JD, Gomes, EB, da Silva, KVL, da Silva,

PKS, \& Fernandes, GP. Brinquedo terapêutico no procedimento de punção venosa: estratégia para reduzir alterações comportamentais. Revista Cuidarte 2016, 7(1), 1163-1170.

18. Soares, VA, Silva, LFD, Santos, PMD, \& Depianti, JRB. A importância do brincar para criança com câncer hospitalizada em cuidado paliativo. Rev. enferm. UFPE on line 2016, 1047-1053 